\title{
Comportamiento coital de adolescentes por características demográficas: Un estudio exploratorio en Ecuador
}

\author{
Adolescent coital behavior by demographic characteristics: An exploratory study in Ecuador
}

\author{
Silvia Guevara-Castro ${ }^{l}$ (D), Elena Jerves-Hermida ${ }^{1,2}$ (D), Ana Cevallos-Neira ${ }^{l}$ (D) \\ 1 Proyecto: Promoción de la Salud Sexual para Adolescentes, "HumSex”, Programa Vlir, Universidad de Cuenca, Cuenca, \\ Ecuador. \\ ${ }^{2}$ Facultad de Filosofía, Letras y Ciencias de la Educación. Universidad de Cuenca, Cuenca-Ecuador. \\ Autora para correspondencia: mdsexologa@gmail.com \\ Fecha de recepción: 26 de febrero de 2019 - Fecha de aceptación: 11 de abril de 2019
}

\section{RESUMEN}

El comportamiento coital del adolescente está constituido por el coito vaginal, oral y anal. Sin embargo, en la literatura, los dos últimos son poco abordados o, en ciertos casos, desconocidos, especialmente en el estudio del comportamiento sexual de adolescentes. Por tal razón, el objetivo de este estudio fue explorar los diferentes tipos de coito en los adolescentes y visibilizar posibles diferencias de acuerdo a factores sociodemográficos: sexo, edad, creencia religiosa, migración parental. La investigación se desarrolló en Cuenca, con una muestra de 2,051 adolescentes escolarizados (15 a 19 años). La muestra se estableció de manera aleatoria. El instrumento utilizado fue el cuestionario autoadministrado "Human Sexuality Questionnaire" (Zucker-man, 1998). Los resultados muestran que el $61 \%$ de los participantes reportó no haber tenido ningún tipo de coito. Entre quienes han tenido coito, el coito vaginal fue el más frecuente (36\%), seguido del coito oral (25\%) y del coito anal (19\%). La frecuencia de coito fue mayor en hombres que en mujeres; pero aumentó en ambos grupos conforme la edad. En mujeres el coito vaginal fue menor entre quienes practican alguna creencia religiosa. La presente investigación evidencia que los adolescentes que expresan haber tenido relaciones coitales, indican haberse involucrado en los diferentes tipos de coito: oral, anal y vaginal; su visualización brinda pautas para entenderlo como parte del desarrollo sexual del adolescente e incluir estos como elementos necesarios en la generación de programas de educación sexual contextualizados.

Palabras clave: Adolescentes, coito, creencia religiosa, migración.

\section{ABSTRACT}

The adolescent's coital behavior is constituted by vaginal, oral and anal intercourse. However, in the literature, the last two are poorly addressed or, in certain cases, unknown, especially in the study of adolescent sexual behavior. For this reason, the objective of this study was to explore the different types of coital behavior in adolescents and to visualize possible differences according to socio-demographic factors: sex, age, religious belief, parental migration. The research was carried out in Cuenca, with a sample of 2051 school-aged adolescents (15 to 19 years old). The sample was randomly established. The instrument used was the self-administered questionnaire "Human Sexuality Questionnaire" (Zuckerman, 1998). The results show that $61 \%$ of the participants reported not having had any type of intercourse. Among those who have had intercourse, vaginal intercourse was the most frequent type (36\%), followed by oral intercourse (25\%) and anal intercourse (19\%). The frequency of intercourse was greater in men than in women; but it increased in both groups according to age. In women, vaginal intercourse was lower among those who practiced some religious belief. The present re-search evidences that adolescents who express having had coital relationships, indicate to have been involved in the different types of intercourse: oral, anal and vaginal; and its visualization provides guidelines to understand it as part of the adolescent's sexual development and to include these as necessary elements in the generation of sex education programs based on their context.
\end{abstract}

Keywords: Adolescents, intercourse, religious practice, migration.

\section{INTRODUCCIÓN}

La sexualidad ha sido entendida como parte esencial del ser humano (Wellings et al., 2006). El repertorio sexual y la diversidad de experiencias sexuales que ocurren en la adolescencia son frecuentes (Halpern-Felsher, Cornell, Kroop, \& Tschann, 2005) y parte del desarrollo positivo integral de la sexualidad (Tolman \& McClelland, 2011). Pese a ello, los programas de educación sexual se han centrado, en su mayoría, en riesgos como el embarazo involuntario y las infecciones de transmisión sexual (Federal Centre for Health Education, 2010).

Esta educación, centrada en elementos preventivos, podría ser explicada por la cantidad de estudios enfocados en los factores de riesgo (Olesen et al., 2011), efectos no deseados producidos por factores familiares, como ausencia del padre (Mendle et al., 2009), uso de sustancias (de Bedout Hoyos, 2010) y el aumento en infecciones de transmisión sexual y embarazo temprano (Rostosky, Regnerus, \& Wright, 2003). Otra particularidad de estos 
estudios es el entendimiento del primer coito como el contacto pene-vagina, dejando de estudiar el coito oral y anal.

La falta de estudios del coito oral y anal puede explicarse por la concepción de que estas actividades no conllevan riesgo de embarazo y mantienen la virginidad en las mujeres (Hal-pern-Felsher, Cornell, Kroop, \& Tschann, 2005). Por ello, incluso se puede explicar la comprensión del coito oral como una actividad no coital (Hensel, Fortenberry, \& Orr, 2008; Olmos, 2011), por ser percibido de menor riesgo frente al coito vaginal (Halpern-Felsher, Cornell, Kroop, \& Tschann, 2005). Contrariamente, el coito anal, por el menor uso de condón, es considerado como una conducta de mayor riesgo (Baldwin \& Baldwin, 2000). Sin embargo, el riesgo de infecciones de transmisión sexual está presente en los tres tipos de coitos (Halpern-Felsher, Cornell, Kroop, \& Tschann, 2005).

Estudios que han abordado el tema del coito difieren significativamente. En Ecuador, el Instituto Nacional de Estadísticas y Censos y el Ministerio de Salud Pública (2012), reportan que el $70.5 \%$ entre los hombres de entre 15 y 24 años han tenido una experiencia sexual, mientras en correspondiente porcentaje en mujeres es de $59.3 \%$, siendo 18.5 años la edad media para la primera relación sexual. Estos datos centran su atención en las mujeres y en el coito vaginal. Este vacío generado respecto al coito oral y anal, repercute en la educación. No abordar estos tipos de coitos, de forma profunda y enfocada desde la búsqueda de sensaciones, deja a la población expuesta al riesgo de infecciones de transmisión sexual (Baldwin \& Baldwin, 2000). No obstante, para contar con programas de educación sexual culturalmente contextualizados y que respondan a los intereses de la sociedad, es necesario conocer las conductas coitales de los adolescentes en cuanto a las variables culturales, de tiempo y grupos sociales; entendiendo el comportamiento sexual del adolescente como algo normativo y sin partir desde un enfoque de riesgo (Tolman \& McClelland, 2011), es decir, desde un enfoque positivo de la sexualidad, en conformidad con la declaración de los Derechos Sexuales (World Association for Sexual Health, 2014).

Es importante considerar el contexto ecuatoriano, en el que la mayoría de la población afirma tener una religión (Instituto Nacional de Estadísticas y Censos, 2012), considerando que la implicación religiosa ha sido asociada con la disminución del involucramiento sexual adolescente (Haglund \& Fehring, 2010). En este sentido, estudios previos que han explorado las visiones parentales, respecto a la sexualidad y la educación sexual en padres de familia de Cuenca, han reportado una visión tradicionalista, por lo que sus intervenciones educativas se basan en la represión y la sanción, fundando sus criterios en principios religiosos y considerando a la sexualidad como una actividad exclusiva de los adultos, en una relación heterosexual y dentro de un matrimonio (Jerves $e t$ al., 2014).

Por otro lado, en Ecuador, durante el año 2000, una importante parte de la población de adultos jóvenes migró fuera del país en búsqueda de oportunidades de trabajo, dejando a hijos pequeños, quienes hoy en día constituyen un grupo importante de adolescentes que se han desarrollado sin la presencia física de sus padres y/o madres. En el Azuay, estudios han reportado que aproximadamente el $35 \%$ de los adolescentes escolarizados de Cuenca tendrían uno o dos padres migrantes (Ron, 2010). Por lo tanto, el objetivo de este estudio fue explorar los diferentes tipos de coito en adolescentes, así como visibilizar posibles diferencias por sexo, edad, creencia religiosa y migración de padres, ya que este conocimiento contribuirá a la difusión de información contextualizada, desde un enfoque de derechos, para que los adolescentes puedan tomar decisiones libres, informadas, voluntarias y responsables sobre su sexualidad, tal como lo estipula la Constitución de la República del Ecuador.

\section{MATERIALES Y MÉTODO}

El estudio es transversal y con un enfoque cuantitativo.

\subsection{Participantes}

El tipo de muestreo fue aleatorio por estratos. La muestra estuvo conformada por 2,051 adolescentes escolarizados provenientes de un universo de 25,870 estudiantes de colegios urbanos y de expansión urbana de la ciudad de Cuenca. La edad de los participantes osciló entre 15 y 19 años $(M=16.12$ años). Los cuestionarios que estuvieron incompletos en más de una tercera parte fueron descartados $(n=72)$. Los datos demográficos mostrados en la Tabla 1 se derivan de un cuestionario auto-administrado.

\subsection{Instrumento}

Para el estudio de la experiencia coital se utilizó una versión traducida al español del "Human Sexuality Questionnaire", de Marvin Zuckerman (1998), de la Universidad de Delaware. El cuestionario consta de 6 subescalas; la escala de medición varía en 5 puntos desde: (1) nunca, (2) una o dos veces, (3) varias veces, (4) más que varias veces, pero menos de 10 y (5) 10 o más veces. El cuestionario fue validado con un nivel de confiabilidad de .921 reportado por el alfa de Cronbach para el conjunto de preguntas estudiadas. Para el presente estudio se describen únicamente los datos referentes al coito. El cuestionario iniciaba con el siguiente enunciado: " $L a$ experiencia sexual es diversa, del listado a continuación, indica si has realizado u otros han realizado la conducta contigo". Las conductas planteadas fueron: "Tus genitales en contacto con la boca" y/o "Tu boca en contacto con los genitales (pene o vulva)" (sexo oral); "Penetración de pene en vagina" (coito vaginal); "Penetración de pene en ano" (coito anal).

Para el análisis del presente estudio, las respuestas fueron dicotomizadas en Sí y No con la finalidad de determinar la experiencia o no del participante en determinado comportamiento coital.

\subsection{Procedimiento}

El cuestionario obtuvo la aprobación del Comité de Ética de la Universidad de Cuenca. El cuestionario fue aplicado a estudiantes de 20 instituciones educativas de la ciudad de Cuenca, seleccionadas aleatoriamente. Para el ingreso a las instituciones educativas físcales se tramitó la respectiva autorización con la Coordinación Zonal de Educación, mientras que para el ingreso a las instituciones particulares la autorización provino del rector(a) de la institución. Posterior a ello, se obtuvo un listado completo de los estudiantes escolarizados y se seleccionaron cursos aleatoriamente para la entrega del consentimiento 
informado; para lo cual hubo el acompañamiento de inspectores o personal del departamento de consejería estudiantil.

Los participantes firmaron el asentimiento informado, se garantizó la confidencialidad y anonimato. La toma de información se realizó en el aula de clase, quienes no tuvieron el consentimiento de parte de sus representantes se abstuvieron de llenar el cuestionario.

\subsection{Análisis estadístico}

Los datos se procesaron utilizando el Software SPSS versión 22. Los resultados se presentan mediante tablas tetracóricas, en las que se muestran las variables aleatorias de coito oral, vaginal y anal, de acuerdo a las variables sociodemográficas estratificadas de acuerdo a sexo y práctica de creencia religiosa. La Tabla 4 fue considerada como variable categórica, misma que está agrupada en cinco años diferentes, por lo tanto, se trata de una variable politómica.

Se ha utilizado el estadístico de prueba Chi-cuadrado de Pearson para el grupo de tablas tetracóricas, mientras que, en el caso de la tabla con una variable politómica, se ha utilizado Chi-cuadrado de homogeneidad. En los resultados de Chi-cuadrado de Pearson se ha complementado la medida con un tamaño del efecto $\varphi$. El valor de la prueba, con sus respectivos grados de libertad $(g l)$, se desglosa en cada tabla.

El nivel de significancia $\alpha$ adoptado para comprobar hipótesis fue de 0.005 y de 0.001 . De obtener un valor menor, se acepta la hipótesis de diferencias en las prácticas coitales, de lo contrario se declara que son iguales.

\section{RESULTADOS}

Tabla 1. Características demográficas de la población la población encuestada. Número total $=2,051$ personas.

\begin{tabular}{cccc}
\hline \multicolumn{2}{c}{ Característica demográfica } & $\mathrm{n}$ & $\%$ \\
\hline \multirow{4}{*}{ Edad } & 15 & 702 & 34.2 \\
& 16 & 639 & 31.2 \\
& 17 & 519 & 25.3 \\
& 18 & 150 & 7.3 \\
Sexo & 19 & 41 & 2.0 \\
\hline Práctica de & Hombres & 936 & 45.6 \\
creencia & Mujeres & 1,115 & 54.4 \\
religiosa & No & 1,445 & 70.5 \\
\hline \multirow{2}{*}{ Migración } & No contesta & 561 & 27.4 \\
de padres & Sí & 564 & 2.2 \\
\hline \multirow{3}{*}{ Migración } & No & 1,444 & 70.4 \\
de & No contesta & 43 & 2.1 \\
progenitor & del padre & 359 & 17.5 \\
& de ambos & 39 & 1.9 \\
& No aplica & 162 & 7.9 \\
& No contesta & 3 & 72.5 \\
\hline
\end{tabular}

La Tabla 1 describe la población de estudio según las características demográficas, y la Tabla 2 describe la frecuencia de coito por sexo. El coito vaginal, estuvo presente en el $50.9 \%(n=467)$ de los hombres, frente al
$23.8 \%(n=262)$ en mujeres. En ambos sexos el coito vaginal fue más frecuente, y la diferencia entre sexos fue estadísticamente significativa $\chi^{2}(1)=159.59, p<.001, \varphi=$ -281. De igual forma, el coito oral estuvo presente en los hombres en un $37.7 \%(n=351)$, mientras que en las mujeres en un $13.7 \%(n=151)$, siendo esta diferencia estadísticamente significativa $\chi^{2}(1)=157.80, p<.001, \varphi$ $=.-278$, sin embargo, con una significación baja e inversa. Finalmente, el coito anal mantiene una frecuencia de $31.6 \%(n=291)$ en hombres, frente al 8.7\% $(n=96)$ en mujeres $\mathrm{y}$, de la misma forma, la diferencia es estadísticamente significativa, pero baja e inversa $\chi^{2}(1)=$ $169.84, p<.001, \varphi=.290$.

La Tabla 3 muestra las diferentes combinaciones de tipos de coito que han tenido los adolescentes según sexo. De la muestra general, el $61 \%(n=1,225)$ no han tenido ningún tipo de coito, de los cuales $45.3 \%(n=412)$ son hombres y 74\% $(n=813)$ son mujeres. Entre quienes han tenido coito, los hombres reportaron mayor frecuencia coital frente a las mujeres. En todas las combinaciones de coito, a excepción de quienes han tenido únicamente coito vaginal -en donde no se visualiza diferencia entre género, fue más frecuente en hombres la combinación de coito oral, vaginal y anal, reportando un $22.9 \%(n=208)$, en tanto que para las mujeres fue más frecuente haber tenido solo coito vaginal, $9.3 \%(n=102)$. El haber tenido coito vaginal y oral se presentó en un $4.6 \%(n=93)$ de la población en general, en tanto el haber tenido únicamente coito oral está representado por $2.2 \%(n=44)$ y el coito anal $0.6 \%(n=12)$, de los cuales $1.1 \%(n=10)$ son hombres y solo $0.2 \%(n=2)$ son mujeres.

La Tabla 4 detalla las conductas coitales según la edad y sexo del adolescente. Se evidenció que, independiente del sexo, las conductas coitales aumentan su frecuencia conforme la edad. El coito vaginal fue la conducta coital más frecuente en cada edad y grupo por sexo; así a los 15 años es referido en $37.4 \%(n=111)$ en hombres y $12.2 \%$ $(n=48)$ en mujeres; porcentaje que aumentó hasta los 19 años, alcanzando un $72.7 \%(n=16)$ en hombres y un 52.9 $\%(n=9)$ en mujeres, resultando estadísticamente significativo bajo y directamente proporcional para hombres $\chi^{2}(4)=52.97, p<.001$, y mujeres $\chi^{2}(4)=93.70$, $p<.001$. En cuanto al coito oral, este aumentó su frecuencia conforme la edad, así su frecuencia en hombres a los 15 años fue de $28.7 \%(n=87)$ llegando a $56.5 \%(n=$ 13) a los 19 años y un nivel de significancia $\chi^{2}(4)=23.13$, $\mathrm{p}<.001$; en tanto en mujeres la frecuencia a los 15 años fue de $8.6 \%(n=34)$ llegando a una frecuencia de $22.2 \%$ $(n=4)$ a los 19 años y una diferencia significativa $\chi^{2}(4)=$ $31.44, p<.001$. Respecto al coito anal, su frecuencia a los 15 años en hombres fue de $24.5 \%(n=73)$ mientras en mujeres fue de $5.9 \%(n=23)$; frecuencia que aumentó hasta $52.2 \%(n=12)$ en hombres, y $11.1 \%(n=2)$ en mujeres a la edad de 19 años. Resultando estadísticamente significativo para hombres $\chi^{2}(4)=28.48, p<.001$, $\mathrm{y}$ mujeres $(4)=18.40, p<.001$.

La Tabla 5 describe la frecuencia de coito, diferenciada por sexo, según practiquen o no alguna creencia religiosa. De esta forma, en los hombres no existió ninguna influencia; mientras sí hubo diferencia en mujeres. Así, entre quienes practican una creencia religiosa, la frecuencia de coito vaginal fue de $20.9 \%(n=167)$, frente a un $32.3 \%(n=91)$ para quienes no practican una creencia religiosa, resultando, aunque baja, esta diferencia significativa y directamente proporcional $\chi^{2}(1)=14.74, p$ $<.001, \varphi=.117$. En cuanto al coito oral, este resultó 
estadísticamente significativo bajo y directamente proporcional $\chi^{2}(1)=4.387, p<.05, \varphi=.064$. Mientras que la relación entre el coito anal y la práctica de creencia religiosa no fue estadísticamente significativa.

La Tabla 6 presenta la frecuencia de coito según migración de padres por sexo, observándose en hombres una frecuencia de coito vaginal de $58.6 \%(n=126)$ en quienes tenían padres migrantes frente a $48.9 \%(n=332)$ en quienes no tenían padres migrantes, con un resultado significativo bajo y directamente proporcional $\chi^{2}(1)=$ $6.161, p<.05, \varphi=.083$. En tanto que, en mujeres, la frecuencia para el coito vaginal resultó estadísticamente no significativa en relación con el tener o no padres migrantes. En lo referente al coito oral y anal, tanto para hombres como para mujeres, no existieron diferencias significativas con respecto a la migración.

Tabla 2. Frecuencia de tipos de coito según el sexo de los encuestados.

\begin{tabular}{|c|c|c|c|c|c|c|c|c|c|}
\hline \multirow{2}{*}{$\begin{array}{l}\text { Tipo de } \\
\text { coito }\end{array}$} & \multirow{2}{*}{ Sexo } & \multicolumn{2}{|c|}{ Sí } & \multicolumn{2}{|c|}{ No } & \multirow[b]{2}{*}{$g l$} & \multirow{2}{*}{$\chi^{2}$} & \multirow[b]{2}{*}{$\varphi$} & \multirow[b]{2}{*}{$p$} \\
\hline & & $n$ & $\%$ & $\mathrm{n}$ & $\%$ & & & & \\
\hline \multirow{3}{*}{ Oral } & Hombres & 351 & 37.7 & 579 & 62.3 & \multirow{3}{*}{1} & \multirow{3}{*}{157.81} & \multirow{3}{*}{-0.278} & \multirow{3}{*}{0.000} \\
\hline & Mujeres & 151 & 13.7 & 955 & 86.3 & & & & \\
\hline & Total & 502 & 24.7 & 1,534 & 75.3 & & & & \\
\hline \multirow{3}{*}{ Vaginal } & Hombres & 467 & 50.9 & 450 & 49.1 & \multirow{3}{*}{1} & \multirow{3}{*}{159.59} & \multirow{3}{*}{-0.281} & \multirow{3}{*}{0.000} \\
\hline & Mujeres & 262 & 23.8 & 839 & 76.2 & & & & \\
\hline & Total & 729 & 36.1 & 1,289 & 63.9 & & & & \\
\hline \multirow{3}{*}{ Anal } & Hombres & 291 & 31.6 & 630 & 68.4 & \multirow{3}{*}{1} & \multirow{3}{*}{169.84} & \multirow{3}{*}{-0.290} & \multirow{3}{*}{0.000} \\
\hline & Mujeres & 96 & 8.7 & 1,006 & 91.3 & & & & \\
\hline & Total & 387 & 19.1 & 1,636 & 80.9 & & & & \\
\hline
\end{tabular}

$g l=$ grados de libertad de la distribución chi-cuadrado; $\chi^{2}=$ estadístico de la prueba chi-cuadrado; $\varphi=$ magnitud del efecto; $p=$ valor-p

Tabla 3. Frecuencia de tipos de coito, y sus combinaciones, según el sexo de los encuestados.

\begin{tabular}{ccccccc}
\hline \multirow{2}{*}{ Tipo de coito } & \multicolumn{2}{c}{ Hombres } & \multicolumn{2}{c}{ Mujeres } & \multicolumn{2}{c}{ Total } \\
\cline { 2 - 7 } & $\mathrm{n}$ & $\%$ & $\mathrm{n}$ & $\%$ & $\mathrm{n}$ & $\%$ \\
\hline Ninguno & 412 & 45.3 & 813 & 74.1 & 1,225 & 61.0 \\
Oral & 24 & 2.6 & 20 & 1.8 & 44 & 2.2 \\
Vaginal & 85 & 9.3 & 102 & 9.3 & 187 & 9.3 \\
Anal & 10 & 1.1 & 2 & 0.2 & 12 & 0.6 \\
Oral y vaginal & 107 & 11.8 & 68 & 6.2 & 175 & 8.7 \\
Oral y anal & 3 & 0.3 & 2 & 0.2 & 5 & 0.2 \\
Vaginal y anal & 61 & 6.7 & 32 & 2.9 & 93 & 4.6 \\
Oral, vaginal y anal & 208 & 22.9 & 58 & 5.3 & 266 & 13.3 \\
\hline Total & 910 & 100 & 1,097 & 100 & 2,007 & 100 \\
\hline
\end{tabular}

Tabla 4. Conductas coitales de acuerdo con la edad y tipo de sexo.

\begin{tabular}{|c|c|c|c|c|c|c|c|c|c|c|c|c|c|c|c|}
\hline \multirow{2}{*}{$\begin{array}{l}\text { Tipo de } \\
\text { coito }\end{array}$} & \multirow{2}{*}{ Edad } & \multicolumn{4}{|c|}{ Hombres } & \multirow{2}{*}{$g l$} & \multirow{2}{*}{$\chi^{2}$} & \multirow[b]{2}{*}{$p$} & \multicolumn{4}{|c|}{ Mujeres } & \multirow{2}{*}{$g l$} & \multirow[b]{2}{*}{$\chi^{2}$} & \multirow[b]{2}{*}{$p$} \\
\hline & & Sí & $\%$ & No & $\%$ & & & & Sí & $\%$ & No & $\%$ & & & \\
\hline \multirow{5}{*}{ Oral } & 15 & 87 & 28.7 & 216 & 71.3 & \multirow{5}{*}{4} & \multirow{5}{*}{23.1} & \multirow{5}{*}{0.001} & 34 & 8.6 & 360 & 91.4 & \multirow{5}{*}{4} & \multirow{5}{*}{31.4} & \multirow{5}{*}{0.001} \\
\hline & 16 & 113 & 37.8 & 186 & 62.2 & & & & 36 & 10.7 & 300 & 89.3 & & & \\
\hline & 17 & 101 & 43.2 & 133 & 56.8 & & & & 58 & 20.7 & 222 & 79.3 & & & \\
\hline & 18 & 37 & 52.1 & 34 & 47.9 & & & & 19 & 24.4 & 59 & 75.6 & & & \\
\hline & 19 & 13 & 56.5 & 10 & 43.5 & & & & 4 & 22.2 & 14 & 77.8 & & & \\
\hline \multirow{5}{*}{ Vaginal } & 15 & 111 & 37.4 & 186 & 62.6 & \multirow{5}{*}{4} & \multirow{5}{*}{53.0} & \multirow{5}{*}{0.001} & 48 & 12.2 & 345 & 87.8 & \multirow{5}{*}{4} & \multirow{5}{*}{93.7} & \multirow{5}{*}{0.000} \\
\hline & 16 & 152 & 51.0 & 146 & 49.0 & & & & 70 & 21.0 & 264 & 79.0 & & & \\
\hline & 17 & 133 & 57.6 & 98 & 42.4 & & & & 92 & 33.0 & 187 & 67.0 & & & \\
\hline & 18 & 55 & 79.7 & 14 & 20.3 & & & & 43 & 55.1 & 35 & 44.9 & & & \\
\hline & 19 & 16 & 72.7 & 6 & 27.3 & & & & 9 & 52.9 & 8 & 47.1 & & & \\
\hline \multirow{5}{*}{ Anal } & 15 & 73 & 24.5 & 225 & 75.5 & \multirow{5}{*}{4} & \multirow{5}{*}{28.5} & \multirow{5}{*}{0.000} & 23 & 5.9 & 370 & 94.1 & \multirow{5}{*}{4} & \multirow{5}{*}{18.4} & \multirow{5}{*}{0.001} \\
\hline & 16 & 91 & 30.6 & 206 & 69.4 & & & & 25 & 7.5 & 310 & 92.5 & & & \\
\hline & 17 & 77 & 33.0 & 156 & 67.0 & & & & 31 & 11.1 & 249 & 88.9 & & & \\
\hline & 18 & 38 & 54.3 & 32 & 45.7 & & & & 15 & 19.7 & 61 & 80.3 & & & \\
\hline & 19 & 12 & 52.2 & 11 & 47.8 & & & & 2 & 11.1 & 16 & 88.9 & & & \\
\hline
\end{tabular}

$g l=$ grados de libertad de la distribución chi-cuadrado; $\chi^{2}=$ estadístico de la prueba chi-cuadrado; $p=$ valor-p 
Tabla 5. Frecuencia de tipos de coitos de acuerdo creencia religiosa y por tipo de sexo.

\begin{tabular}{|c|c|c|c|c|c|c|c|c|c|c|}
\hline \multirow{2}{*}{ Sexo } & \multirow{2}{*}{$\begin{array}{l}\text { Tipo de } \\
\text { coito }\end{array}$} & \multirow{2}{*}{$\begin{array}{l}\text { Creencia } \\
\text { religiosa } \\
\end{array}$} & \multicolumn{2}{|c|}{ Sí } & \multicolumn{2}{|c|}{ No } & \multirow{2}{*}{$g l$} & \multirow{2}{*}{$\chi^{2}$} & \multirow[b]{2}{*}{$\varphi$} & \multirow[b]{2}{*}{$p$} \\
\hline & & & $\mathrm{n}$ & $\%$ & $\mathrm{n}$ & $\%$ & & & & \\
\hline \multirow{6}{*}{ Hombres } & \multirow{2}{*}{ Oral } & Sí & 237 & 37.4 & 369 & 62.6 & \multirow{2}{*}{1} & \multirow{2}{*}{0.085} & \multirow{2}{*}{0.010} & \multirow{2}{*}{0.771} \\
\hline & & No & 105 & 38.5 & 168 & 61.5 & & & & \\
\hline & \multirow{2}{*}{ Vaginal } & Sí & 318 & 50.9 & 307 & 49.1 & \multirow{2}{*}{1} & \multirow{2}{*}{0.001} & \multirow{2}{*}{-0.001} & \multirow{2}{*}{0.971} \\
\hline & & No & 136 & 50.7 & 132 & 49.3 & & & & \\
\hline & \multirow{2}{*}{ Anal } & Sí & 195 & 31.2 & 430 & 68.8 & \multirow{2}{*}{1} & \multirow{2}{*}{0.054} & \multirow{2}{*}{0.008} & \multirow{2}{*}{0.816} \\
\hline & & No & 87 & 32.0 & 185 & 68.0 & & & & \\
\hline \multirow{6}{*}{ Mujeres } & \multirow{2}{*}{ Oral } & Sí & 99 & 12.3 & 703 & 87.7 & \multirow{2}{*}{1} & \multirow{2}{*}{4.387} & \multirow{2}{*}{0.064} & \multirow{2}{*}{0.036} \\
\hline & & No & 49 & 17.3 & 134 & 82.7 & & & & \\
\hline & \multirow{2}{*}{ Vaginal } & Sí & 167 & 20.9 & 631 & 79.1 & \multirow{2}{*}{1} & \multirow{2}{*}{14.743} & \multirow{2}{*}{0.117} & \multirow{2}{*}{0.000} \\
\hline & & No & 91 & 32.3 & 191 & 67.7 & & & & \\
\hline & \multirow{2}{*}{ Anal } & Sí & 62 & 7.8 & 738 & 92.3 & 1 & & & 0065 \\
\hline & & No & 32 & 11.3 & 250 & 88.7 & 1 & 12 & 0 & 0.003 \\
\hline
\end{tabular}

$\overline{g l}=$ grados de libertad de la distribución chi-cuadrado; $\chi^{2}=$ estadístico de la prueba chi-cuadrado; $\varphi=$ magnitud del efecto; $p=$ valor-p.

Tabla 6. Frecuencia de tipos de coitos de acuerdo con la migración de padres y por tipo de sexo.

\begin{tabular}{|c|c|c|c|c|c|c|c|c|c|c|}
\hline \multirow{2}{*}{ Sexo } & \multirow{2}{*}{$\begin{array}{l}\text { Tipo de } \\
\text { coito }\end{array}$} & \multirow{2}{*}{$\begin{array}{c}\text { Migración } \\
\text { de padres }\end{array}$} & \multicolumn{2}{|c|}{ Sí } & \multicolumn{2}{|c|}{ No } & \multirow{2}{*}{$g l$} & \multirow{2}{*}{$\chi^{2}$} & \multirow[b]{2}{*}{$\varphi$} & \multirow[b]{2}{*}{$p$} \\
\hline & & & $\mathrm{n}$ & $\%$ & $\mathrm{n}$ & $\%$ & & & & \\
\hline \multirow{6}{*}{ Hombres } & \multirow{2}{*}{ Oral } & Sí & 89 & 40.5 & 131 & 59.5 & \multirow{2}{*}{1} & \multirow{2}{*}{0.655} & \multirow{2}{*}{0.027} & \multirow{2}{*}{0.418} \\
\hline & & No & 257 & 37.4 & 430 & 62.6 & & & & \\
\hline & \multirow{2}{*}{ Vaginal } & Sí & 126 & 58.6 & 89 & 41.4 & \multirow{2}{*}{1} & \multirow{2}{*}{6.161} & \multirow{2}{*}{0.083} & \multirow{2}{*}{0.013} \\
\hline & & No & 332 & 48.9 & 347 & 51.1 & & & & \\
\hline & \multirow{2}{*}{ Anal } & Sí & 74 & 34.1 & 143 & 65.9 & \multirow{2}{*}{1} & \multirow{2}{*}{0.887} & \multirow{2}{*}{0.031} & \multirow{2}{*}{0.346} \\
\hline & & No & 143 & 65.9 & 472 & 69.3 & & & & \\
\hline \multirow{6}{*}{ Mujeres } & \multirow{2}{*}{ Oral } & Sí & 43 & 12.6 & 297 & 87.4 & \multirow{2}{*}{1} & \multirow{2}{*}{0.405} & \multirow{2}{*}{-0.019} & \multirow{2}{*}{0.525} \\
\hline & & No & 105 & 14.1 & 641 & 85.9 & & & & \\
\hline & \multirow{2}{*}{ Vaginal } & Sí & 84 & 24.9 & 253 & 75.1 & \multirow{2}{*}{1} & \multirow{2}{*}{0.358} & \multirow{2}{*}{0.018} & \multirow{2}{*}{0.549} \\
\hline & & No & 173 & 23.3 & 571 & 76.7 & & & & \\
\hline & \multirow{2}{*}{ Anal } & Sí & 27 & 8.0 & 311 & 92.0 & 1 & 0231 & 5 & 0 \\
\hline & & No & 66 & 8.9 & 678 & 91.1 & 1 & 0.201 & -0.015 & 0.031 \\
\hline
\end{tabular}

$\overline{g l}=$ grados de libertad de la distribución chi-cuadrado; $\chi^{2}=$ estadístico de la prueba chi-cuadrado; $\varphi=$ magnitud del efecto; $p=$ valor-p.

\section{DISCUSIÓN}

En este estudio se usó la aleatorización de los estudiantes de los colegios de la ciudad de Cuenca para poder generalizar los resultados de la conducta sexual a los estudiantes de la zona urbana de la ciudad. El objetivo de la investigación estuvo centrado en conocer los diferentes tipos de coito presentes en los adolescentes de Cuenca según sus factores sociodemográficos. Este objetivo fue elegido porque el foco de estudios sobre el comportamiento sexual de los adolescentes está usualmente centrado en el coito vaginal (Song \& HalpernFelsher, 2011); existiendo por tanto un escaso conocimiento local acerca de la variación de la conducta sexual y según los factores demográficos. De esta manera, al tener en cuenta las diferencias demográficas del grupo social, los datos obtenidos corresponden al contexto de los participantes, para, a partir de estas conductas, entender el comportamiento normativo típico de los adolescentes (Tolman \& McClelland, 2011).

A continuación, se realiza un análisis de los datos obtenidos, en relación con el coito vaginal, oral y anal, los cuales son presentados por edad y sexo, creencia religiosa y estructura familiar, agrupados bajo los factores demográficos estudiados, con lo cual se puede tener una mayor claridad del impacto de estos en el comportamiento sexual adolescente.

\subsection{Edad y sexo}

La actividad sexual en hombres y mujeres inicia en la adolescencia tardía, es decir, entre los 15 y 19 años (Wellings et al., 2006), lo cual se puede visualizar también con el incremento del coito conforme a la edad. Sin embargo, el inicio de la actividad sexual es referenciado, generalmente, al coito vaginal, existiendo diferencias de edad según los diferentes países. Así, el inicio de la actividad sexual reportado en Chile es de 17 años, mientras en Perú y Brasil es de 16.5 años (Wellings et al., 2006).

Al hablar por edades, en Brasil, a los 15 años cerca de $40 \%$ de los hombres y $11 \%$ de las mujeres tienen experiencia de coito vaginal (Gray \& García, 2013), lo cual concuerda con los datos del presente estudio, en el cual $37 \%$ de hombres y $12 \%$ de mujeres indicaron haber tenido coito vaginal a esta edad. Además, conforme avanza la edad del adolescente aumenta el porcentaje de frecuencia del coital vaginal, así, en el presente estudio, a la edad de 16 años la mitad de los adolescentes hombres han tenido coito vaginal, aumentando a un $80 \%$ a los 18 años, concordando estos datos con los estudios de Halpern \& Haydon (2012) y Gates \& Sonenstein (2000), quienes refieren que las dos 
terceras partes de los adolescentes han tenido coito vaginal a esta edad. Pero, esta concordancia está dada solo para el grupo de varones, pues en mujeres la frecuencia de coito vaginal a los 18 años es de 55\%.

En cuanto al coito oral, comparado con el coito vaginal, su frecuencia aumenta conforme la edad, pero, a la vez, mantiene una frecuencia menor a este, concordando con Halpern \& Haydon (2012) y evidenciando la diferencia de frecuencias coitales según la región y contexto social. Por ejemplo, los adolescentes de Estados Unidos perciben al coito oral de menor riesgo y más aceptable que el coito vaginal (Halpern-Felsher, Cornell, Kroop, \& Tschann, 2005), lo cual explica su aparición antes que el coito vaginal (Song \& Halpern-Felsher, 2011). Sin embargo, esta situación no se da en el presente estudio, con lo cual se podría descartar la idea del coito oral como estrategia para conservar la virginidad (Remez, 2000 citado en Tolman \& McClelland, 2011).

Respecto al coito anal, la frecuencia aumenta conforme la edad, manteniendo las diferencias por sexo. A su vez, la frecuencia de coito anal en el presente estudio muestra que tres de cada diez hombres han tenido coito anal, lo cual es superior a los resultados obtenidos por Leichliter, Chandra, Liddon, Fenton, \& Aral (2007) y Halpern \& Haydon (2012), quienes refieren que uno de cada diez adolescentes -hombres y mujeres- ha tenido coito anal a los 18 años, coincidente con el porcentaje encontrado en este estudio en el grupo de mujeres.

Respecto a las conductas coitales a la edad de 19 años, Gates \& Sonenstein (2000), reportan que en Estados Unidos más de la mitad de la población ha tenido coito oral, vaginal o anal; en tanto, Hensel, Fortenberry \& Orr (2008), reportan una frecuencia de $38 \%$ para quienes han tenido coito vaginal con otra conducta coital y $4.7 \%$ para quienes han tenido coito vaginal, oral y anal. El presente estudio no mostró concordancia con ninguno de estos estudios. Así, en promedio, en el grupo de 15 a 19 años, alrededor del $13 \%$ de la población tiene los tres tipos de experiencia coital (oral, vaginal y anal).

Las conductas coitales según sexo mostraron diferencia entre hombres y mujeres (Part, Rahu, Rahu, \& Karro, 2011). De igual forma, Wellings et al., (2006) encontraron diferencias de género más pronunciadas en países menos industrializados, lo cual concuerda con la mayor prevalencia de conductas coitales reflejadas en hombres frente a mujeres. En el presente estudio, representando una diferencia de 2 a 1 en el coito vaginal, en tanto en el coito oral y coito anal esta diferencia fue de 3 a 1 .

Esta diferencia de conductas coitales entre sexos puede ser explicada desde la teoría de aprendizaje social cognitivo de Bandura, la cual considera que las diferencias conductuales de género son creadas por la observación de otros al ser reforzadas o castigadas (Mills \& Bukowski, 2013). Estas conductas son internalizadas como estándares apropiados de género y regulan la conducta acorde a la norma de género. Dicha teoría podría explicar la mayor frecuencia coital de los hombres frente a las mujeres de su misma edad.

\subsection{Religión}

En Ecuador, según el último censo del Instituto Nacional de Estadísticas y Censos (2012), el 91.95\% de la población indicó profesar alguna religión; en este sentido, tomando en cuenta que, generalmente, la religión es asociada con la prohibición del sexo premarital, el presente estudio indagó respecto a si los participantes practican alguna creencia religiosa, respuesta que fue analizada en relación con el tipo de coito.

Algunos estudios han encontraron asociación entre la religión y el retardo en el inicio de actividades coitales (Hull, Hennessy, Bleakley, Fishbein, \& Jordan, 2011; Rostosky, Regnerus, \& Wright, 2003). Esto es explicado por el reforzamiento de la ideología sobre lo prohibido o negativo del coito en la adolescencia (Rostosky, Regnerus, \& Wright, 2003), siendo asociado a las mujeres, por el énfasis en la virginidad, así como a su mayor participación en la iglesia (Burdette \& Hill, 2009). Los resultados de este estudio sugieren que el énfasis en la virginidad en mujeres, pero no en hombres, podría ser una explicación a la asociación de mujeres y la ausencia de una asociación en hombres entre la práctica de la creencia religiosa y el coito vaginal. Con respecto al coito oral y coito anal, estudios previos no han encontrado ninguna asociación entre este tipo de comportamientos coitales y la religión (Burdette \& Hill, 2009; Baldwin \& Baldwin, 2000); situación concordante con los datos del presente estudio. Estos hallazgos podrían estar relacionados con la falta de discusión del líder religioso, al igual que la definición de relaciones referidas únicamente de forma implícita al coito vaginal (Burdette \& Hill, 2009).

\subsection{Estructura familiar}

La estructura familiar ha sido relacionada previamente con el inicio sexual temprano de adolescentes (Cavazos-Rehg et al., 2010). De esta forma, la ausencia de los padres, así como de la supervisión y apoyo (Cavazos-Rehg et al., 2010) y el menor involucramiento con sus hijos (Coley, Votruba-Drzal, \& Schindler, 2009) es relacionado con la iniciación sexual temprana. Por estos datos, y debido a la fragmentación familiar física producto de desplazamientos familiares, producida por la migración en la provincia del Azuay (Pedone, 2010) se indagó acerca de si su padre, madre o ambos habían migrado. Los resultados obtenidos mostraron una asociación baja en hombres para el coito vaginal, pero no hubo asociación para hombres o mujeres para el coito oral y anal; lo cual puede explicarse por la calidad de paternidad, además de la estructura familiar (Manlove, Wildsmith, Ikramullah, Terry-Humen, \& Schelar, 2012). Otra explicación puede ser debido a las nuevas dinámicas de reconstitución de lazos familiares, así como el mantenimiento de relaciones familiares a distancia, conocida como familia transnacional (Herrera, 2004). De hecho, estudios previos sugieren que en las familias transnacionales los vínculos con miembros de la familia extendida podrían constituir un importante elemento de estabilidad para el desarrollo del adolescente (Jerves, De Heane, Enzlin, \& Rober, 2016).

\section{CONCLUSIONES}

Los adolescentes de ambos sexos se involucran en diferentes tipos de coito: vaginal, oral y anal. Los resultados señalados tienen implicaciones para la intervención en educación sexual basada en la evidencia, la cual debería incluir una enseñanza de todos los tipos de prácticas sexuales coitales, es decir, se debe incluir la enseñanza de prácticas coitales orales y anales, a pesar de no ser tan frecuentes como el coito vaginal, pues forman parte del repertorio coital del adolescente, quien, al no 
recibir información certera queda expuesto a factores de riesgo. Es momento de dejar de evitar estos temas y preguntarse acerca de las habilidades y cambios esperados en los adolescentes para posicionarlos como parte del desarrollo sexual positivo (Tolman \& McClelland, 2011).

\section{AGRADECIMIENTOS}

Este estudio contó con el financiamiento del Programa de Cooperación Universitaria Institucional (IUC) entre la Universidad de Cuenca (Ecuador) y las Universidades Flamencas, a través del Consejo Interuniversitario Flamenco (VLIR-UOS), y del GAD Municipal del Cantón Cuenca a través de la Red de Salud Sexual y Salud Reproductiva del Cantón Cuenca.

\section{REFERENCIAS}

Asamblea Constituyente. (2008). Constitución de la República del Ecuador. Quito.

Baldwin, J. I., \& Baldwin, J. D. (2000). Heterosexual anal intercourse: an understudied, high-risk sexual behavior. Archives of sexual behavior, 29(4), 357-373. doi: 10.1023/A:1001918504344

Burdette, A. M., \& Hill, T. D. (2009). Religious involvement and transitions into adolescent sexual activities. Sociology of Religion, 70(1), 28-48. doi: 10.1093/socrel/srp011

Cavazos-Rehg, P. A., Spitznagel, E. L., Bucholz, K. K., Nurnberger, J. J., Edenberg, H. J., Kramer, J. R., et al. (2010). Predictors of sexual debut at age 16 or younger. Archives of sexual behavior, 39(3), 664-673. doi: 10.1007/s10508-008-9397-y

Coley, R. L., Votruba-Drzal, E., \& Schindler, H. S. (2009). Fathers' and mothers' parenting predicting and responding to adolescent sexual risk behaviors. Child development, 80(3), 808-827. Recuperado de http://onlinelibrary.wiley.com/doi/10.1111/j.14678624.2009.01299. $\mathrm{x} /$ abstract;jsessionid=AB599BD8730 62E21D8B4E33ECCDCD0BA.f01t03?userIsAuthentic ated $=$ false $\&$ deniedAccessCustomisedMessage $=$

de Bedout Hoyos, A. (2010). Asociación entre consumo de sustancias y relaciones sexuales en adolescentes estudiantes en Vegachí, Antioquia, Colombia. Revista de Psicología GEPU, 1(2), 31-38. Recuperado de https://dialnet.unirioja.es/descarga/articulo/4392183.pd $\mathrm{f}$

Federal Centre for Health Education. (2010). Standars for sexuality education in Europe. Recuperado de https://www.oif.ac.at/fileadmin/OEIF/andere_Publikati onen/WHO_BZgA_Standards.pdf

Gates, G. J., \& Sonenstein, F. L. (2000). Heterosexual genital sexual activity among adolescent males: 1988 and 1995. Family planning perspectives, 295-304. doi: $10.2307 / 2648198$

Gray, P. B., \& García, J. R. (2013). Evolution and human sexual behavior. Cambridge: Harvard University Press.

Haglund, K. A., \& Fehring, R. J. (2010). The association of religiosity, sexual education, and parental factors with risky sexual behaviors among adolescents and young adults. Journal of Religion and Health, 49(4), 460-472.

Halpern, C. T., \& Haydon, A. A. (2012). Sexual timetables for oral-genital, vaginal, and anal intercourse: Sociodemographic comparisons in a nationally representative sample of adolescents. American Journal of Public Health, 102(6), 1221-1228. doi: 10.2105/AJPH.2011.300394

Halpern-Felsher, B. L., Cornell, J. L., Kroop, R. Y., \& Tschann, J. M. (2005). Oral versus vaginal sex among adolescents: Perceptions, attitudes, and behavior. Pediatrics, 115(4), 845-851. doi: 10.1542/peds.20042108

Hensel, D. J., Fortenberry, J. D., \& Orr, D. P. (2008). Variations in coital and noncoital sexual repertoire among adolescent women. Journal of Adolescent Health, 42(2), 170-176. doi: 10.1016/j.jadohealth.2007.07.009

Herrera, G. (2004). Elementos para una comprensión de las familias transnacionales desde la experiencia migratoria del Sur del Ecuador. En F. Hidalgo, Migraciones: Un juego con cartas marcadas (págs. 215-231). Quito: ILDIS-Abya Yala. Recuperado de http://repository.unm.edu/bitstream/handle/1928/11767 /Migraciones\%20un\%20juego\%20con\%20cartas.pdf?s equence $=1 \#$ page $=213$

Hull, S. J., Hennessy, M., Bleakley, A., Fishbein, M., \& Jordan, A. (2011). Identifying the causal pathways from religiosity to delayed adolescent sexual behavior. Journal of sex research, 48(6), 543-553. doi: 10.1080/00224499.2010.521868

Instituto Nacional de Estadísticas y Censos. (2012, agosto). Primeras estadísticas oficiales sobre filiación religiosa en el Ecuador. Recuperado de http://www.ecuadorencifras.gob.ec//documentos/webinec/Estadisticas_Sociales/Filiacion_Religiosa/presenta cion_religion.pdf

Instituto Nacional de Estadísticas y Censos; Ministerio de Salud Pública. (2012). Encuesta Nacional de Salud y Nutrición- ENSANUT 2012. Demografía, salud materna e infantil y salud sexual y reproductiva. Recuperado de

http://www.ecuadorencifras.gob.ec/salud-saludreproductiva-y-nutricion/

Jerves, E., De Heane, L., Enzlin, P., \& Rober, P. (2016). Adolescents' lived experiences of close relationships in the context of transnational families: A qualitative study from Ecuador. Journal of Adolescent Research.

Jerves, E., López, S., Castro, C., Ortiz, W., Palacios, M., Rober, P., et al. (2014). Understanding parental views of adolescent sexuality and sex education in Ecuador: a qualitative study. Sex Education, 14(1), 14-27.

Leichliter, J. S., Chandra, A., Liddon, N., Fenton, K. A., $\&$ Aral, S. O. (2007). Prevalence and correlates of heterosexual anal and oral sex in adolescents and adults in the United States. Journal of Infectious Diseases, 196(12), 1852-1859. doi: 10.1086/522867

Manlove, J., Wildsmith, E., Ikramullah, E., TerryHumen, E., \& Schelar, E. (2012). Family environments and the relationship context of first adolescent sex: Correlates of first sex in a casual versus steady relationship. Social science research, 41(4), 861-875. doi: 10.1016/j.ssresearch.2012.02.003 
Mendle, J., harden, K. P., Turkheimer, E., Van Hulle, C. A., D’Onofrio, B. M., Brooks-Gunn, J., et al. (2009). Associations between father absence and age of first sexual intercourse. Child development, 80(5), 1463 1480. doi: $10.1111 /$ j. 1467

Mills, K. D., \& Bukowski, W. M. (2013). Sexual Development. In D. S. Bromberg, \& W. T. O’Donohue, Handbook of Child and Adolescent Sexuality (pp. 115144). London: Elsevier.

Olesen, T. B., Jensen, K. E., Nygård, M., Tryggvadottir, L., Sparén, P., Hansen, T. B., et al. (2011). oung age at first intercourse and risk-taking behaviours - a study of nearly 65000 women in four Nordic countries. The European Journal of Public Health, 22(2), 220-224. doi: 10.1093/eurpub/ckr055

Olmos, J. L. (2011). Mujeres que no han tenido relaciones sexuales: consideraciones sobre la conducta Toko-ginecología práctica, (719), 189-198.

Part, K., Rahu, K., Rahu, M., \& Karro, H. (2011). Gender differences in factors associated with sexual intercourse among Estonian adolescents. Scandinavian journal of public health, 39(4), 389-395. doi: $10.1177 / 1403494810395820$

Pedone, C. (2010). "Varones aventureros" vs. "madres que abandonan": reconstrucción de las relaciones familiares a partir de la migración ecuatoriana. Revista Interdisciplinar da Mobilidade Humana, 16(30). Recuperado de http://www.csem.org.br/remhu/index.php/remhu/article /view/76
Ron, S. (2010). Impacto de la migración en la salud mental en niños y adolescentes, Cuenca 2010. Universidad de Cuenca. Facultad de Ciencias Médicas. Escuela de Medicina, 87-88.

Rostosky, S. S., Regnerus, M. D., \& Wright, M. L. (2003). Coital debut: The role of religiosity and sex attitudes in the Add Health Survey. Journal of sex research, 40(4), 358-367. Recuperado de http://www.tandfonline.com/doi/abs/10.1080/00224490 209552202

Song, A. V., \& Halpern-Felsher, B. L. (2011). Predictive relationship between adolescent oral and vaginal sex: Results from a prospective, longitudinal study. Archives of pediatrics \& adolescent medicine, 165(3), 243-249. doi:10.1001/archpediatrics.2010.214

Tolman, D. L., \& McClelland, S. I. (2011). Normative sexuality development in adolescence: A decade in review, 2000-2009. Journal of Research on Adolescence, 21(1), 242-255. doi: 10.1111/j.15327795.2010.00726.x

Wellings, K., Collumbien, M., Slaymaker, E., Sing, S., Hodges, Z., Patel, D., et al. (2006). Sexual behaviour in context: a global perspective. The Lancet, 368(9548), 1706-1728. http://dx.doi.org/10.1016/S01406736(06)69479-8

World Association for Sexual Health. (2014). Declaration of Sexual Rights. Recuperado de http://www.worldsexology.org/wpcontent/uploads/2013/08/declaration_of_sexual_rights_ sep03_2014.pdf 